\title{
Aggravation of Hemorrhoids in Patients With Cervical Cancer Undergoing Radiotherapy: A Nationwide Population-based Study
}

\author{
INCHEOL SEO ${ }^{1}$ and HYUNSOO JANG ${ }^{2}$ \\ Departments of ${ }^{1}$ Microbiology and ${ }^{2}$ Radiation Oncology, School of Medicine, \\ Dongguk University, Gyeongju, Republic of Korea
}

\begin{abstract}
Background/Aim: Anal canal toxicity tends to be ignored in pelvic radiotherapy $(R T)$. However, patients with hemorrhoids can be troubled by lower radiation dose. We tried to determine whether a correlation exists between hemorrhoids and anal symptoms in patients with cervical cancer undergoing RT. Patients and Methods: The insurance claim data of patients who underwent definitive treatment for cervical cancer from 2015 to 2019 were analyzed. Adverse events including bleeding, proctitis, and hemorrhoids, were documented for 1 year after treatment completion. Odds ratios (ORs) were estimated by unconditional Poisson regression and adjusted for age, treatments, chemotherapy, and comorbidities. Results: Details of 67,114 insured cervical cancer patients treated between 2015 and 2019 were obtained. Among them, 5,919 patients with follow-up data for at least one year, treated with curative intent, were analyzed. The OR of the definitive radiotherapy group (DRT group) for anal bleeding was 10.57 higher than that of the operation alone group (surgical group) $(p<0.01)$. Newly developed hemorrhoids gradually increased in the surgical group $(3.17 \%)$, the postoperative radiotherapy group (5.38\%), and the DRT group (7.58\%). The OR of the DRT group for newly developed hemorrhoids was 2.38 higher than that of the surgical group $(p<0.01)$, and ORs increased to 1.99 and 1.61 in patients that received chemotherapy and patients with diabetes, respectively $(p<0.01)$. Conclusion: Pelvic $R T$ increased anal bleeding and symptomatic hemorrhoids. In
\end{abstract}

This article is freely accessible online.

Correspondence to: Hyunsoo Jang, Department of Radiation Oncology, Dongguk University School of Medicine, Gyeongju, Republic of Korea. Tel: +82 547708552, Fax: +82 547708554 e-mail: opencagejhs@gmail.com

Key Words: Cervical cancer, hemorrhoids, radiotherapy, radiation proctitis. particular, chemotherapy and diabetes also increased the risk. If patients with hemorrhoids receive pelvic $R T$, attention is required to prevent hemorrhoid aggravation.

Radiotherapy (RT) is an important treatment option for cervical cancer $(1,2)$. RT is an effective treatment, but sideeffects of adjacent normal organs are inevitable. Radiation proctitis is a typical side-effect associated with pelvic RT (3, 4). In particular, in patients with hemorrhoids, RT may aggravate anal symptoms such as pain or bleeding $(5,6)$. In general, radiation proctitis tolerance is around $50 \mathrm{~Gy}$ (7). But, anal symptoms occur at lower doses in patients with hemorrhoids and these symptoms may be due to hemorrhoid aggravation rather than radiation proctitis $(5,8)$. Until now, hemorrhoid aggravation by pelvic RT has not been a topic of research interest, and thus, a substantial number of hemorrhoid aggravations may have been attributed to radiation proctitis. We considered that if this is the case, anal symptoms caused by pelvic RT can be reduced by proper hemorrhoid management.

Anal canal toxicity tends to be a neglected side-effect of pelvic RT. The anal canal is known to tolerate prescribed whole-pelvic RT doses of 45-50 Gy (7). However, in patients with hemorrhoids, since the outer wall of the anal canal is weak, there is a possibility that hemorrhoids may worsen at lower doses $(5,6)$. Furthermore, only a small number of studies have examined the relationship between hemorrhoid aggravation and RT dose $(5,8,9)$. However, the risk of aggravation increases further when internal hemorrhoids are considered, and patients with asymptomatic hemorrhoids are likely to develop symptomatic hemorrhoids after RT.

From a treatment point of view, differentiating radiationinduced proctitis and hemorrhoid aggravation may not be meaningful. However, it may be valuable from the view point of prevention. In this study, we investigated the relationship between hemorrhoids and anal symptoms in patients with cervical cancer that received RT using the database of the Health Insurance Review \& Assessment Service (HIRA). In 
Table I. Characteristics of the study population.

\begin{tabular}{|c|c|c|c|c|c|c|c|c|}
\hline \multirow[b]{2}{*}{ No. of cases (\% total) } & \multicolumn{2}{|c|}{ Total } & \multicolumn{2}{|c|}{ DRT } & \multicolumn{2}{|c|}{ PRT } & \multicolumn{2}{|c|}{ Surgery alone } \\
\hline & 5,919 & $(100.00 \%)$ & 1,513 & $(25.56 \%)$ & 1,691 & $(28.57 \%)$ & 2,715 & $(45.87 \%)$ \\
\hline Age $($ mean $\pm S D)$ & 52.33 & $(12.92)$ & 58.27 & $(14.03)$ & 50.38 & (11.54) & 50.24 & (12.03) \\
\hline \multicolumn{9}{|l|}{ Comorbidities } \\
\hline Hypertension & 2,159 & $(36.48 \%)$ & 739 & $(48.84 \%)$ & 588 & $(34.77 \%)$ & 832 & $(30.64 \%)$ \\
\hline Diabetes & 1,811 & $(30.60 \%)$ & 557 & $(36.81 \%)$ & 525 & $(31.05 \%)$ & 729 & $(26.85 \%)$ \\
\hline Dyslipidemia & 3,795 & $(64.12 \%)$ & 1,014 & $(67.02 \%)$ & 1,081 & $(63.93 \%)$ & 1,700 & $(62.62 \%)$ \\
\hline Hemorrhoid before Tx & 212 & $(3.58 \%)$ & 51 & $(3.37 \%)$ & 75 & $(4.44 \%)$ & 86 & $(3.17 \%)$ \\
\hline \multicolumn{9}{|l|}{ RT type } \\
\hline Conformal RT & 1,831 & $(30.93 \%)$ & 1,001 & $(66.16 \%)$ & 830 & $(49.08 \%)$ & 0 & $(0.00 \%)$ \\
\hline IMRT & 1,344 & $(22.71 \%)$ & 512 & $(33.84 \%)$ & 832 & $(49.20 \%)$ & 0 & $(0.00 \%)$ \\
\hline Chemotherapy & 2,924 & $(49.40 \%)$ & 1,373 & $(90.75 \%)$ & 1,317 & $(77.88 \%)$ & 234 & $(8.62 \%)$ \\
\hline
\end{tabular}

DRT: Definitive radiotherapy; PRT: postoperative radiotherapy; SD: standard deviation; RT: radiotherapy; IMRT: intensity modulated radiotherapy.

addition, risk factors associated with treatment modalities, RT planning techniques, and underlying diseases were analyzed.

\section{Patients and Methods}

Korea has a public insurance system called the National Health Insurance (NHI) system, which covers almost all residents, and data such as sex, age, diagnosis, prescriptions, and procedures for nearly all medical claims in South Korea are stored by HIRA. The present study was conducted using nationwide data from the HIRA database. The study subjects were patients diagnosed with cervical cancer between January 2015 and December 2019. Patients with distant metastasis or a concurrent malignancy at the time of cervical cancer diagnosis were excluded. The study was approved beforehand by the institutional review board (IRB) of Dongguk University Gyeongju Hospital (IRB no. 110757-202004-HR-03-02).

The study population consisted of patients diagnosed with a malignant neoplasm of cervix uteri (C53), endocervix (C53.0), exocervix (C53.1), overlapping lesion of cervix uteri (C53.8), or an unspecified lesion of cervix uteri (C53.9) according to the Korean Classification of Disease, $6^{\text {th }}$ edition (KCD-6), which is a version of the International Classification of Disease 10 (ICD-10), modified for the Korean health care system. Adverse events including bleeding (K62.5, K62.6), proctitis (K62.7, K6.28), and hemorrhoid (K64, K64.0, K64.1, K64.2, K64.8, K64.9) were documented for 1 year after treatment completion. To detect hemorrhoid aggravation caused by radiation, we only analyzed symptoms occurring over the year following RT because although RT may have effects beyond one year, we considered these effects would probably involve other factors.

Patients were identified by procedure codes for 3D-conformal RT (3D-CRT) (HD061), intensity-modulated RT (IMRT) (HZ271), and intracavitary therapy (HD081, HD082). Patients that underwent definitive surgery were identified using procedure codes for simple hysterectomy (R0141, R0142, R4143, R4144, R4147, R4148), or radical hysterectomy (R4154, R4155). Subjects that survived less than 1 year after treatment were excluded from the analysis.

Odds ratio (OR) and their 95\% confidence intervals (CI) were estimated by unconditional Poisson regression and adjusted for age, treatment type, chemotherapy, and comorbidities. The statistical analysis was performed with $\mathrm{R}$ version 3.4.2.

\section{Results}

Details of 67,114 patients treated for cervical cancer from 2015 to 2019 were obtained from the HIRA database, and the data of 5,919 patients treated with curative intent with at least one year of follow-up data were analyzed. Median patient age was 52 years, and 2,715 patients underwent surgery only (surgical group), 1,513 underwent definitive radiotherapy (DRT group), and 1,691 underwent postoperative radiotherapy (PRT group). IMRT planning was performed for $42 \%$ of the patients that received RT. Chemotherapy was administered to 2,924 patients. Detailed patient characteristics are provided in Table I.

$R T$ increased anal bleeding and proctitis. After treatment, anal bleeding was increased by RT or chemotherapy. The OR of anal bleeding was 10.57 higher in the DRT group than in the surgical group and 2.69 higher than in the PRT group. Patients who received chemotherapy had an OR of 3.07. Proctitis exhibited a similar pattern; the OR was 52.08 higher in the DRT group than in the surgical group and 3.35 higher than in the PRT group. Patients who received chemotherapy had an OR of 7.10. Multivariate analysis showed the DRT group had significantly higher ORs for anal bleeding and proctitis. Details are presented in Tables II and III.

Newly diagnosed hemorrhoids increased after RT. It is difficult to differentiate hemorrhoids and proctitis based on considerations of symptoms alone. We analyzed newly diagnosed hemorrhoids that developed within 1 year of RT. A total of 291 patients were newly diagnosed with hemorrhoids and the median age of affected patients was 52 . The incidence rate gradually increased in the surgical group (3.17\%), PRT group (5.38\%), and DRT group (7.58\%). No difference was observed between RT planning techniques, 
Table II. Crude and adjusted odds ratios for bleeding after treatments.

\begin{tabular}{|c|c|c|c|c|c|c|}
\hline & \multicolumn{3}{|c|}{ Univariate } & \multicolumn{3}{|c|}{ Multivariate } \\
\hline & OR & $95 \% \mathrm{CI}$ & $p$-Value & OR & $95 \% \mathrm{CI}$ & $p$-Value \\
\hline Age & 1.0084 & $(0.9919-1.0247)$ & 0.3140 & 0.9713 & $(0.9532-0.9897)$ & 0.0024 \\
\hline \multicolumn{7}{|c|}{ Treatment (ref=DRT) } \\
\hline PRT & 0.3714 & $(0.2215-0.6018)$ & 0.0001 & 0.3092 & $(0.1782-0.5190)$ & 0.0000 \\
\hline Surgery alone & 0.0946 & $(0.0436-0.1823)$ & $<0.0001$ & 0.0607 & $(0.0237-0.1469)$ & $<0.0001$ \\
\hline Chemotherapy & 3.0728 & $(1.9088-5.1533)$ & $<0.0001$ & 0.6303 & $(0.3445-1.2200)$ & 0.1504 \\
\hline \multicolumn{7}{|l|}{ Comorbidities } \\
\hline Hypertension & 2.2122 & $(1.4409-3.4218)$ & 0.0003 & 1.8219 & $(1.0991-3.0299)$ & 0.0202 \\
\hline Diabetes & 1.7857 & $(1.1545-2.7417)$ & 0.0083 & 1.2914 & $(0.7963-2.0892)$ & 0.2974 \\
\hline Dyslipidemia & 2.0522 & $(1.2467-3.5587)$ & 0.0069 & 1.7321 & $(1.0066-3.1090)$ & 0.0548 \\
\hline
\end{tabular}

DRT: Definitive radiotherapy; PRT: postoperative radiotherapy; OR: odds ratio; CI: confidence interval.

Table III. Crude and adjusted odds ratios for bleeding after treatments.

\begin{tabular}{|c|c|c|c|c|c|c|}
\hline & \multicolumn{3}{|c|}{ Univariate } & \multicolumn{3}{|c|}{ Multivariate } \\
\hline & OR & $95 \% \mathrm{CI}$ & $p$-Value & OR & $95 \% \mathrm{CI}$ & $p$-Value \\
\hline Age & 1.0287 & $(1.0151-1.0424)$ & $<0.0001$ & 0.9963 & $(0.9808-1.0122)$ & 0.6480 \\
\hline \multicolumn{7}{|c|}{ Treatment (ref=DRT) } \\
\hline PRT & 0.2982 & $(0.1927-0.4481)$ & $<0.0001$ & 0.3218 & $(0.2035-0.4951)$ & $<0.0001$ \\
\hline Surgery alone & 0.0192 & $(0.0047-0.0512)$ & $<0.0001$ & 0.0256 & $(0.0058-0.0797)$ & $<0.0001$ \\
\hline Chemotherapy & 7.1017 & $(4.2712-12.7104)$ & $<0.0001$ & 1.2936 & $(0.7288-2.4772)$ & 0.4063 \\
\hline \multicolumn{7}{|l|}{ Comorbidities } \\
\hline Hypertension & 2.3221 & $(1.6186-3.3524)$ & $<0.0001$ & 1.4334 & $(0.9366-2.2025)$ & 0.0984 \\
\hline Diabetes & 1.9494 & $(1.3560-2.7933)$ & 0.0003 & 1.3004 & $(0.8690-1.9458)$ & 0.2007 \\
\hline Dyslipidemia & 1.9071 & $(1.2606-2.9838)$ & 0.0032 & 1.4561 & $(0.9263-2.3513)$ & 0.1126 \\
\hline
\end{tabular}

DRT: Definitive radiotherapy; PRT: postoperative radiotherapy; OR: odds ratio; CI: confidence interval.

and the incidence of newly diagnosed hemorrhoids in the chemotherapy group was about twice that in the nonchemotherapy group. In addition, hemorrhoid incidences were higher in patients with diabetes, hypertension, and dyslipidemia (Table IV).

Risk factors of newly diagnosed hemorrhoids after RT. The OR of newly diagnosed hemorrhoids within 1 year after RT was 2.38 higher in the DRT group than in the surgical group and 1.40 higher than in the PRT group. Patients that received chemotherapy and patients with diabetes had ORs of 1.99 and 1.61, respectively. In multivariate analysis, DRT and diabetes showed a statistically significant OR (Table V).

\section{Discussion}

This study was undertaken to determine whether RT aggravates hemorrhoids in patients with cervical cancer. We found that RT significantly increased rates of hemorrhoid aggravation and anal bleeding in this patient population. The
Table IV. Newly diagnosed hemorrhoids during the 12 months following RT.

\begin{tabular}{llcc}
\hline & & \multicolumn{2}{c}{ Hemorrhoid } \\
\hline Total & & 291 & $(4.92 \%)$ \\
Mean age (SD) & & 52.46 & $(13.16)$ \\
Treatments (\% total) & DRT & 114 & $(7.53 \%)$ \\
& PRT & 91 & $(5.38 \%)$ \\
& Surgery alone & 86 & $(3.17 \%)$ \\
RT types & Total & 204 & $(6.43 \%)$ \\
& Conformal RT & 116 & $(6.34 \%)$ \\
Chemotherapy & IMRT & 88 & $(6.55 \%)$ \\
& Chemotherapy+ & 192 & $(6.57 \%)$ \\
Comorbidities & Chemotherapy- & 99 & $(3.31 \%)$ \\
& Hypertension+ & 120 & $(5.56 \%)$ \\
& Hypertension- & 171 & $(4.55 \%)$ \\
& Diabetes+ & 121 & $(6.68 \%)$ \\
& Diabetes- & 170 & $(4.14 \%)$ \\
& Dyslipidemia+ & 197 & $(5.19 \%)$ \\
& Dyslipidemia- & 94 & $(4.43 \%)$ \\
& & & \\
& & &
\end{tabular}

DRT: Definitive radiotherapy; PRT: postoperative radiotherapy; SD: standard deviation; RT: radiotherapy; IMRT: intensity modulated radiotherapy. 
in vivo $35: 2357-2361(2021)$

Table V. Crude and adjusted odds ratios for newly developed hemorrhoids after treatment.

\begin{tabular}{|c|c|c|c|c|c|c|}
\hline & \multicolumn{3}{|c|}{ Univariate } & \multicolumn{3}{|c|}{ Multivariate } \\
\hline & OR & $95 \% \mathrm{CI}$ & $p$-Value & OR & $95 \% \mathrm{CI}$ & $p$-Value \\
\hline Age & 1.0008 & $(0.9919-1.0097)$ & 0.8610 & 0.9883 & (0.9780-0.9987) & 0.0273 \\
\hline \multicolumn{7}{|c|}{ Treatment (ref=DRT) } \\
\hline PRT & 0.7142 & $(0.5412-0.9399)$ & 0.0167 & 0.7001 & $(0.5242-0.9323)$ & 0.0151 \\
\hline Surgery alone & 0.4204 & $(0.3170-0.5554)$ & $<0.0001$ & 0.5190 & $(0.3387-0.7937)$ & 0.0026 \\
\hline Chemotherapy & 1.9865 & $(1.5631-2.5404)$ & $<0.0001$ & 1.3401 & (0.9296-1.9528) & 0.1225 \\
\hline \multicolumn{7}{|l|}{ Comorbidities } \\
\hline Hypertension & 1.2221 & $(0.9659-1.5414)$ & 0.0921 & 1.0516 & $(0.7961-1.3854)$ & 0.7218 \\
\hline Diabetes & 1.6145 & $(1.2765-2.0358)$ & 0.0001 & 1.6314 & $(1.2493-2.1267)$ & 0.0003 \\
\hline Dyslipidemia & 1.1730 & (0.9204-1.5055) & 0.2030 & 1.0268 & $(0.7852-1.3490)$ & 0.8478 \\
\hline
\end{tabular}

DRT: Definitive radiotherapy; PRT: postoperative radiotherapy; OR: odds ratio; CI: confidence interval.

anorectal side-effects of pelvic irradiation are classified as radiation-induced proctitis (10), and the effect of irradiation on hemorrhoids is often neglected. As a result, few studies have addressed the relationship between RT and hemorrhoids $(5,8,9)$. The symptoms of acute proctitis are diarrhea and tenesmus and those of chronic proctitis are bleeding and painful defecation (10). However, if hemorrhoids are exacerbated, bleeding and pain appear early in the RT period $(5,6)$. Therefore, the differential diagnosis of hemorrhoid aggravation and radiation-induced proctitis is essential to ensure that proper treatment choices are made, and it should be determined whether acute anal symptoms are due to hemorrhoid aggravation or lower rectal wall problems. If it is decided that symptoms are due to hemorrhoid aggravation, clinical management involving sitz baths and dietary changes is necessary $(11,12)$. Although acute anal symptoms are not considered severe, once exacerbated, they may be painful for long periods and result in poor tolerance to RT. For these reasons, patients with hemorrhoids need to be managed at RT initiation.

It is known that tolerable dose for anal canal and rectum is around 50-60 Gy $(7,13)$, but it has been reported that hemorrhoid aggravation occurs at doses around $30 \mathrm{~Gy}$ (5), which indicates radiation-induced proctitis and hemorrhoid aggravation have different tolerance doses. When wholepelvic RT is performed at total doses not exceeding $50 \mathrm{~Gy}$, asymptomatic hemorrhoids are often ignored clinically or addressed with empirical anal blocks, although recent advances in RT techniques enable more sophisticated types of RT, such as IMRT, to be used. 3D-CRT using box techniques has been widely used for postoperative RT in cervical cancer. Several recent research studies have used pelvic IMRT to reduce radiation exposure to normal tissues such as bladder, rectum, and small bowel (14-16). However, the anus is infrequently considered an organ at risk. In this study, we compared the effects of 3D-CRT and IMRT on hemorrhoid aggravation and bleeding frequency, but no significant differences were observed between the two modalities. We recommend that more detailed analysis be conducted to investigate the effects of RT techniques, total doses, and fractioned doses. Unfortunately, since this study was conducted using insurance claim data, detailed data on RT schedules were not obtained.

Many factors cause hemorrhoids such as age, low-fiber diet, pregnancy, weight, underlying diseases, and bowel habits $(12,17,18)$. To identify the effect of RT on hemorrhoids, we analyzed rates of anal bleeding and newly diagnosed hemorrhoids within one year after RT. We found that the ORs of both were significantly higher in the DRT group than in the surgical group. It is reasonable that asymptomatic hemorrhoids were worsened by RT, rather than the occurrence of new hemorrhoids. Although the number of newly diagnosed hemorrhoid cases was only 291 among 5,919 patients, this study was based on insurance claim data, and thus, only hemorrhoids that required active treatment were included. There is a study showing that the location of anal verge and anal canal has a large deviation for each patient (8). Patients have different anatomical structures and different underlying diseases. Therefore, different treatments are required for each patient. In particular, patients with hemorrhoids can be affected by small amounts of radiation, and thus, careful attention is needed from the treatment planning stage. For example, if a patient with hemorrhoid and diabetes receives pelvic irradiation, the anal canal dose should be reduced and strict diabetes control is required. Diet or medication may be modified to prevent constipation.

As this study was based on insurance claim codes, specific information about RT schedules (e.g., on planning techniques and radiation doses) and detailed information on hemorrhoid 
and toxicity severities were not available. However, the study was performed using the data of a large number of patients, and thus, we believe that our findings are statistically meaningful. Notably, the study shows RT worsens anal bleeding and hemorrhoids. In particular, patients with diabetes and those that undergo combined chemotherapy are at an increased risk. If patients with hemorrhoids receive pelvic RT, careful management is required to prevent hemorrhoid aggravation. We recommend that additional studies be performed to determine the effects of different RT schedules and techniques on hemorrhoid aggravation.

\section{Conflicts of Interest}

The Authors declare that they have no competing interests for this study.

\section{Authors' Contributions}

IS and HJ contributed equally to conception, design, acquisition of data, and wrote the manuscript.

\section{References}

1 Eifel PJ, Winter K, Morris M, Levenback C, Grigsby PW, Cooper J, Rotman M, Gershenson D and Mutch DG: Pelvic irradiation with concurrent chemotherapy versus pelvic and paraaortic irradiation for high-risk cervical cancer: an update of radiation therapy oncology group trial (RTOG) 90-01. J Clin Oncol 22(5): 872-880, 2004. PMID: 14990643. DOI: 10.1200/JCO.2004.07.197

2 Rose PG, Bundy BN, Watkins EB, Thigpen JT, Deppe G, Maiman MA, Clarke-Pearson DL and Insalaco S: Concurrent cisplatin-based radiotherapy and chemotherapy for locally advanced cervical cancer. N Engl J Med 340(15): 1144-1153, 1999. PMID: 10202165. DOI: 10.1056/NEJM199904153401502

3 Mansha MA, Sadaf T, Waheed A, Munawar A, Rashid A and Chaudry SJ: Long-term toxicity and efficacy of intensitymodulated radiation therapy in cervical cancers: experience of a cancer hospital in Pakistan. JCO Glob Oncol 6: 1639-1646, 2020. PMID: 33112682. DOI: 10.1200/GO.20.00169

4 Huang EY, Lin H, Wang CJ, Chanchien CC and Ou YC: Impact of treatment time-related factors on prognoses and radiation proctitis after definitive chemoradiotherapy for cervical cancer. Cancer Med 5(9): 2205-2212, 2016. PMID: 27416796. DOI: $10.1002 /$ cam 4.794

5 Jang H, Baek JG and Yoo SJ: Acute anal toxicity after whole pelvic radiotherapy in patients with asymptomatic haemorrhoids: identification of dosimetric and patient factors. Br J Radiol 88(1050): 20150022, 2015. PMID: 25873480. DOI: 10.1259/ bjr.20150022

6 Peng X, Zhou S, Liu S, Li J, Huang S, Jiang X, Lin M, Huang $\mathrm{S}$, Lin C, Qian C, Liu M and He L: Dose-volume analysis of predictors for acute anal toxicity after radiotherapy in prostate cancer patients. Radiat Oncol 14(1): 174, 2019. PMID: 31601249. DOI: 10.1186/s13014-019-1374-1
7 Moreau-Claeys MV and Peiffert D: [Normal tissue tolerance to external beam radiation therapy: anal canal]. Cancer Radiother 14(4-5): 359-362, 2010. PMID: 20418146. DOI: 10.1016/ j.canrad.2010.01.009

8 Jang H, Baek JG and Jo S: The anal canal as a risk organ in cervical cancer patients with hemorrhoids undergoing whole pelvic radiotherapy. Tumori 101(1): 72-77, 2015. PMID: 25702647. DOI: $10.5301 / \mathrm{tj} .5000219$

9 Baek JG, Kim EC, Kim SK and Jang H: Dosimetric planning study for the prevention of anal complications after postoperative whole pelvic radiotherapy in cervical cancer patients with hemorrhoids. Br J Radiol 88(1056): 20150223, 2015. PMID: 26395671. DOI: 10.1259/bjr.20150223

10 Babb RR: Radiation proctitis: a review. Am J Gastroenterol 91(7): 1309-1311, 1996. PMID: 8677984.

11 Mott T, Latimer K and Edwards C: Hemorrhoids: diagnosis and treatment options. Am Fam Physician 97(3): 172-179, 2018. PMID: 29431977.

12 Sandler RS and Peery AF: Rethinking What we know about hemorrhoids. Clin Gastroenterol Hepatol 17(1): 8-15, 2019. PMID: 29601902. DOI: 10.1016/j.cgh.2018.03.020

13 Blanchard P and Chapet O: [Normal tissue tolerance to external beam radiation therapy: rectum]. Cancer Radiother 14(4-5): 354358, 2010. PMID: 20570201. DOI: 10.1016/j.canrad. 2010.02 .012

14 Tharavichitkul E, Wanwilairat S, Chakrabandhu S, Klunklin P, Onchan W, Tippanya D, Nopnop W, Galalae R and Chitapanarux I: Image-guided brachytherapy (IGBT) combined with whole pelvic intensity-modulated radiotherapy (WP-IMRT) for locally advanced cervical cancer: a prospective study from Chiang Mai University Hospital, Thailand. J Contemp Brachytherapy 5(1): 10-16, 2013. PMID: 23634150. DOI: 10.5114/jcb.2013.34338

15 Erpolat OP, Alco G, Caglar HB, Igdem S, Saran A, Dagoglu N, Aslay I, Ozsaran Z, Demirci S, Keven E, Guney Y, Akmansu M, Kilic D, Bayman E, Etiz D and Mandel NM: Comparison of hematologic toxicity between 3DCRT and IMRT planning in cervical cancer patients after concurrent chemoradiotherapy: a national multi-center study. Eur J Gynaecol Oncol 35(1): 62-66, 2014. PMID: 24654465.

16 Chang Y, Yang ZY, Li GL, Li Q, Yang Q, Fan JQ, Zhao YC, Song YQ and $\mathrm{Wu}$ G: Correlations between radiation dose in bone marrow and hematological toxicity in patients with cervical cancer: a comparison of 3DCRT, IMRT, and RapidARC. Int J Gynecol Cancer 26(4): 770-776, 2016. PMID: 26844613. DOI: 10.1097/IGC.0000000000000660

17 Riss S, Weiser FA, Schwameis K, Riss T, Mittlböck M, Steiner $\mathrm{G}$ and Stift $\mathrm{A}$ : The prevalence of hemorrhoids in adults. Int $\mathrm{J}$ Colorectal Dis 27(2): 215-220, 2012. PMID: 21932016. DOI: 10.1007/s00384-011-1316-3

18 Fox A, Tietze PH and Ramakrishnan K: Anorectal conditions: hemorrhoids. FP Essent 419: 11-19, 2014. PMID: 24742083.
Received April 2, 2021

Revised April 14, 2021

Accepted April 16, 2021 